\title{
MECANIZACIÓN AGRÍCOLA Y FOMENTO ESTATAL EN CHILE (1942-1973)
}

\section{AGRARIAN MECHANIZATION AND STATE SUPPORT IN CHILE (1942-1973)}

\author{
Andrés Rojas Böttner \\ Universidad Autónoma de Chile, Santiago de Chile, Chile, arojas@ichem.cl
}

\begin{abstract}
Resumen. Frente al rezago del sector agrícola, a partir de la década de 1940 el Estado en Chile asumió como tarea el fomento de la mecanización de faenas, creando para ello una serie de instituciones. Tras tres décadas, a pesar del aumento significativo del parque nacional el déficit no pudo ser revertido, manteniéndose la necesidad de importar grandes volúmenes de alimento. El artículo analiza el impacto de las políticas estatales de fomento a la mecanización, describiendo la evolución del parque en el ámbito nacional y regional, considerando para ello el contexto productivo y las condiciones en que se produjo la incorporación de maquinaria. Finalmente, se profundiza en la participación del Estado a partir del caso del Servicio de Equipos Agrícolas Mecanizados (SEAM), órgano creado en específico para la materia.
\end{abstract}

Palabras clave: mecanización agrícola; fomento estatal; Servicio de Equipos Agrícolas Mecanizados; Chile.

Abstract. Faced with the lag in the agrarian sector, from the 1940s onwards, the state of Chile embarked on the task of promoting the mechanization of agrarian chores, creating a series of institutions to that end. After three decades, despite the significant expansion of the national productive apparatus, the government had failed to halt the deficit, meaning that the need to import large quantities of food remained. The article analyzes the impact of state policies of promoting mechanization, describing the evolution of the productive apparatus on a national and regional level, and, to this end, considering the context production and the conditions under which the incorporation of machinery took place. Finally, the article analyzes state participation on the basis of the case of the Mechanized Agricultural Equipment Service (Servicio de Equipos Agrícolas Mecanizados, SEAM), a body created specifically for this purpose.

Key words: agricultural mechanization; state support; Mechanized Agricultural Equipment Service; Chile.

Fecha de recepción: 3 de agosto de 2015. Fecha de aceptación: 18 de enero de 2016.

Am. Lat. Hist. Econ., sep.-dic., 2016, pp. 147-173 | DOI: 10.18232/alhe.v23i3.720 


\section{INTRODUCCIÓN}

partir de la década de 1930 el sector agrícola en Chile comenzó a A mostrar síntomas de rezago. La crisis del modelo primario expor1 tador y de los mercados internacionales evidenció un sector poco modernizado y con bajos rendimientos productivos, que en las décadas siguientes no fue capaz de satisfacer la demanda interna de alimentos, aumentando el déficit y los volúmenes de importación. Frente a esto, surgieron varias interpretaciones sobre sus causas. Una primera corriente se centró en la estructura de las explotaciones agrarias del país, apuntando a la marcada concentración de la propiedad de la tierra y al régimen laboral tradicional que imperaba en las haciendas, lo que sería causa de baja productividad y subocupación de las tierras cultivables disponibles (Ahumada, 1958; Corporación para la Reforma Agraria, 1970; Pinto, 1959). Otras interpretaciones enfatizaron la baja rentabilidad del sector derivada de la fijación de precios, bajo un umbral que incentivase la inversión productiva. Esta postura interpretaba que la agricultura estaba siendo sacrificada en pos del desarrollo industrial (Almonacid, 2009; Mamalakis, 1976; Santana, 2006). El foco del debate finalmente giró en torno al tema de la reforma agraria y la sindicalización campesina, clivaje político desde mediados de siglo (Avendaño, 2014).

Mayor consenso hubo en la necesidad de mecanizar las faenas para aumentar la producción, aunque con matices en torno a los ritmos de implementación (por el impacto en el empleo agrícola) y al financiamiento. En consecuencia, el Estado asumió la tarea de fomentar la mecanización agrícola, generando en las tres décadas siguientes una institucionalidad para esos fines. En el presente artículo se aborda el proceso de mecanización agrícola con fomento estatal entre los años 1942 y 1973, periodo en el cual se desarrolla una institucionalidad y se ejecutan las principales políticas en la materia. Las fuentes predominantes en este estudio corresponden a informes (tanto de circulación pública como restringida) realizados por instituciones del Estado, tesis universitarias del periodo, estadísticas, censos agropecuarios, legislación y bibliografía secundaria.

\section{ANTECEDENTES DEL SECTOR AGRÍCOLA EN CHILE}

Desde mediados del siglo XIX la agricultura en Chile se caracterizó por la fuerte expansión de los cultivos cerealeros, en especial del trigo, desplazando en importancia al sector ganadero. Dicha expansión estuvo impulsada por la demanda externa derivada de las fiebres del oro en Australia y California, y posteriormente por los mercados internos del norte minero y 
las zonas urbanas. Se trataba de una actividad extensiva, amparada en los bajos costos de la mano de obra y la fuerte concentración de la propiedad de la tierra. A su vez, se caracterizaba por la baja inversión en bienes de capital y el uso mayoritario de herramientas tradicionales como el arado de palo y el uso de la tracción animal, en especial de bueyes (menos aptos que los caballos) (Bauer, 1994; Mc Bride, 1938).

El aumento de la demanda de alimentos durante el ciclo del salitre (1883- ca. 1930), sumado a la migración laboral hacia las calicheras del norte, no lograron provocar un efecto de intensificación productiva a gran escala, siendo cubierta la mayor demanda principalmente por la incorporación del espacio agrícola de La Frontera, territorio de ocupación indígena al sur del río Biobío (Santana, 2006). Con ello, el área de vocación agrícola incorporó decenas de miles de hectáreas, abarcando principalmente los valles (depresión intermedia) de las zonas central y sur, de climas mediterráneos y subhúmedos, con suelos de gran fertilidad. ${ }^{1}$ Dicho de otro modo, en general se profundizó en el uso extensivo gracias al stock ampliado de tierras, siendo excepcional la mecanización dada en algunos cultivos en territorios cercanos a las grandes ciudades (Bengoa, 1990; Robles, 2003). Salvo por el proyecto de un grupo de socios de internar maquinaria a través de la Sociedad Nacional de Agricultura (SNA) -con efectos limitados por la oposición interna y la cantidad de recursos destinados (Robles, 2009) - no hubo una orientación a la mecanización productiva. ${ }^{2}$ Varios factores actuaban como desincentivo a la modernización.

En primer lugar, la estructura laboral del campo. En 1952 la población rural representaba 48.7\% de la población total (Dirección General de Estadística, 1953) y 30\% de la fuerza de trabajo se desempeñaba en el área agrícola, en condiciones precarias y bajo una débil legislación laboral rural, negociada políticamente a cambio de los intereses de trabajadores urbanos e industriales (Gómez, 2004; Moulian, 2006). Los salarios agrícolas, inferiores a las otras ramas productivas -especialmente los jornaleros y afuerinos- tendieron a estancarse y divergir aún más a partir de la segunda mitad de la década de 1950 (Rodríguez, 2013).

En segundo lugar, la internación de maquinaria significaba un alto costo, no sólo por el equipo en sí mismo, sino que también por los costos

${ }^{1}$ Las mayores excepciones la constituyen el suelo aluvial de la posición alta de la zona central (franco arenoso) y el granítico de la costa de la misma zona (franco arenoso), ambas con menos de $2 \%$ de materia orgánica (Letelier, 1967, p. 71). Dadas estas condiciones, en comparación con otros países, el uso de fertilizantes en Chile fue relativamente bajo (17.14 kg/ha en 1962), aunque por sobre el promedio de América Latina (p. 21).

${ }^{2}$ La SNA era el principal gremio de los grandes productores agrícolas. En el periodo considerado se interesó principalmente en materias de subsidios, seguros y aranceles; uso de fertilizantes y selección de semillas, y oposición a las reformas laborales en el campo (Carrière, 1981; Correa, 2005). 
del flete y la internación aduanera, aunque a partir de 1950 se eximió de aranceles la internación de maquinaria agrícola (Ley núm. 9.839, art. 14). Si se consideraba el flete, el almacenaje y los seguros, además de la máquina, el valor se incrementaba por sobre 40\% (CORFO, 1969b, p. 40). Una vez asumidos estos costos, el funcionamiento de un tractor con un complemento (incluyendo el combustible) representaba alrededor de 37\% del costo de una explotación modernizada, apenas por debajo del costo de la mano de obra (40\%) (Meller, 1998). Se debe agregar además la carencia de personal calificado, tanto para el manejo como para la reparación de la maquinaria. En consecuencia, los costos eran muy difíciles de asumir para la mayor parte de las explotaciones, que no contaban con capital suficiente para ello.

A mediados de la década de 1930, ya iniciada la crisis, las unidades mayores (sobre $2000 \mathrm{ha}$ ) representaban 68\% de las tierras agrícolas, estando en manos de menos de $1 \%$ de los propietarios. En contraste, las unidades menores (menos de $20 \mathrm{ha}$ ) representaban 3\% de las tierras agrícolas y estaban controladas por $73 \%$ de los propietarios. ${ }^{3}$ A nivel territorial la concentración se encontraba más acentuada en la zona sur, en donde la media de extensión de las explotaciones era el doble de la zona central (142 y 77 ha respectivamente).

Los principales cultivos a nivel país, considerando la superficie cultivada en el año agrícola 1935-1936, fueron el trigo blanco (53\%), la avena $(6 \%)$, los frijoles $(5 \%)$, la cebada cervecera $(5 \%)$, el trigo candeal $(4 \%)$, las papas (3\%) y el maíz (3\%). Como ha sido anticipado, considerando el total de hectáreas cultivadas, el núcleo agrícola principal fueron las zonas central $(46 \%)$ y sur $(43 \%)$, muy distantes de las zonas norte $(11 \%)$ y austral (menos de 1\%). Con las características señaladas, el sector agrícola debió enfrentar la crisis del modelo primario exportador, cerrando un ciclo de relativo auge y entrando a una etapa de rezago. De manera sintética, Bengoa (1990) afirma:

Las consecuencias las conocemos: despoblamiento y migraciones campo-ciudad, decaimiento de la producción agrícola y creciente proceso de importación de alimentos a partir de la postguerra, atraso rural generalizado. A partir de la crisis del año 30 se inició un periodo de decadencia latifundiaria, que culminaría con la Reforma Agraria. El origen de este proceso se encuentra en las modernizaciones y cambios no realizados en el periodo anterior de prosperidad (p. 210).

\footnotetext{
${ }^{3}$ Para este cálculo se consideraron las propiedades con explotación primaria y secundaria (se excluyeron las propiedades sin explotación) y con indicación de superficie (Dirección General de Estadística, 1938).
} 
Indicadores del rezago señalado son, por ejemplo, que entre las décadas de 1940 y 1970 el crecimiento anual del sector agrícola fue de sólo $1.9 \%$ (prácticamente igual que la población), distante del $4.3 \%$ del producto geográfico bruto (excluido el sector agrícola), mostrando a su vez una tasa decreciente de generación de empleo $(-0.4 \%$ anual $)$ y una productividad que se mantuvo apenas en un tercio respecto del promedio de los otros sectores, provocando que en las décadas de 1950 y 1960 la importación de alimentos representara cerca de 30\% del déficit comercial (Meller, 1998).

En ese contexto adverso, el Estado intervino a partir de una serie de medidas de distinto tipo, ya fuese en cuanto a la comercialización, a través de la fijación de precios y poderes de compra, como en cuanto a la producción, principalmente a través de la mecanización de las faenas. A continuación se presenta de manera global el marco de intervención del Estado para posteriormente profundizar en los planes de mecanización implementados.

\section{EsTADO Y MECANIZACIÓN AGRÍCOLA EN CHILE}

El Estado ha sido un promotor importante de la mecanización agraria en varios países. ${ }^{4}$ En el caso de Chile, con posterioridad a la década de 1930, se produjeron algunos cambios importantes, especialmente en cuanto a su rango de acción. En el contexto del prestigio de las ideas de planificación económica (Muñoz y Arriagada, 1977), especialmente a partir del triunfo del centroizquierdista Frente Popular en 1938, el Estado asumió una nueva participación en materias sociales y económicas. Dentro de estas últimas estuvo la promoción de la modernización agrícola, mantenida (con matices) por los distintos gobiernos (Millar y Fernández, 2008). Para dicha tarea, se fue creando de manera gradual una institucionalidad encabezada por el Ministerio de Agricultura ${ }^{5}$ y la Corporación de Fomento de la Producción (en adelante CORFO).

El primero, como secretaría de Estado, estaba encargado de la política agraria, de la investigación, orientación y coordinación de la producción agropecuaria (Urzúa y García, 1971). Contaba con atribuciones importantes para intervenir en el mercado de los alimentos, como la fijación de precios, el establecimiento de poderes compradores, cuotas de importación y

\footnotetext{
${ }^{4}$ Para el caso argentino, véanse Barsky (1994) y Raccanello (2012); para el mexicano, véanse Negrete (2014) y Cepeda y Gitli (1985), y para algunos casos de Europa continental, véase Fernández (1999). En casos como el peruano, la promoción del Estado se centró en otras medidas de fomento, como la irrigación (Figallo y Vattuone, 1989).

${ }^{5}$ En 1924 fue creado el Ministerio de Agricultura, Industria y Colonización. Posteriormente, en 1930 quedó definido como cartera exclusiva para la agricultura.
} 
exportación, etc. (Gómez, 1944), atribuciones que resultaron determinantes en los desempeños de los distintos territorios, como se profundizará luego. Durante el periodo en estudio, de gran expansión estatal, fue uno de los ministerios que menos creció (apenas se duplicó, llegando a 1284 funcionarios), lo que es un claro reflejo de la pérdida de importancia relativa de la agricultura para el Estado. Más bien, el Ministerio de Agricultura fue reorganizado. Las principales instituciones creadas en el periodo fueron el Instituto de Desarrollo Agropecuario en 1960, centrado en el fortalecimiento de los pequeños y medianos agricultores, y la Corporación para la Reforma Agraria en 1963, encargada de coordinar los procesos de expropiación y repartición de tierras entre los campesinos. ${ }^{6}$

La segunda, creada en 1939, fue la principal agencia estatal impulsora del proceso de industrialización y modernización del país, a través de la cual se diseñaban y ejecutaban ambiciosos planes de inversión pública y políticas de fomento productivo y coordinación multisectorial (con énfasis en la producción industrial) (Ortega, 1989). De la CoRfo se derivó una serie de organismos, algunos de los cuales se transformaron en empresas autónomas del Estado, donde se definieron las principales medidas para la mecanización agrícola.

En 1940 la corporación presentó un plan de acción inmediata, en el cual uno de los objetivos fue la mecanización de labores agrícolas. Inicialmente se aspiró a la fabricación parcial de la maquinaria en Chile, importando el resto con apoyo de créditos a largo plazo por intermedio de la Caja de Crédito Agrario, las Sociedades Agrícolas y firmas comerciales (CORFO, 1940). Sin embargo, la opción de producción nacional sólo prosperó en el caso de los equipamientos más simples, como las rastras y los pulverizadores manuales. La idea reapareció un par de décadas después, nuevamente sin resultados significativos (Oficina de Planificación Nacional, 1970).

En 1940 la CoRfo y la Caja Agraria ${ }^{7}$ iniciaron sus operaciones de crédito e importación de maquinaria, a baja escala los primeros años por problemas causados por la segunda guerra mundial (Ortega, 1989). En 1946 se elaboró un programa de mecanización que dio una importancia casi exclusiva a la internación de equipos. Así, en 1950 se decretó la exención arancelaria para la internación de maquinaria agrícola (Ley núm. 9.839,

${ }^{6}$ Ambas instituciones fueron traspasadas al área descentralizada en 1964 (sacadas del Ministerio de Agricultura), favoreciendo su despliegue territorial (Urzúa y García, 1971, pp. 108-110).

${ }^{7}$ La fusión de la Caja de Crédito Agrario, la Caja de Crédito Hipotecario, la Caja Nacional de Ahorro y el Instituto de Crédito Industrial dieron origen al Banco del Estado de Chile en 1953, institución relevante en el financiamiento de la mecanización en Chile. 
art. 14). ${ }^{8}$ Sin embargo, no se consideraron en la medida los repuestos, aun-

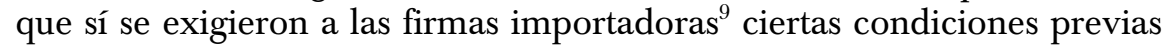
respecto a atención mecánica, reparación y manutención de los equipos. Lo anterior no fue suficiente, convirtiéndose en un problema grave, incluso después de 1962, en que se facultó al presidente de la república para decretar exención arancelaria a los repuestos (Ley núm. 15.020, art. 54). El bajo impacto de la medida se debió a su aplicación en forma restringida, debido a la constatación del desvío de las piezas importadas hacia otros sectores productivos (CORFO, 1969a).

A mediados de la década de 1950 se presentó el Plan de Desarrollo Agrícola y de Transportes (publicado en 1954), hoja de ruta para el desarrollo del agro, el cual estaba basado en las recomendaciones del informe La Economía Agrícola de Chile (1952).$^{10}$ Ese plan estableció para el periodo 1954-1957 la meta de importación de 1460 tractores anuales (4 670000 dólares estadunidenses) y un mismo monto para el resto de maquinaria agrícola, y para 1958-1961, 2250 tractores anuales (7 200000 dólares estadunidenses) y un mismo monto para el resto de maquinaria agrícola.

En total, el plan consideraba la importación de 14840 tractores por un monto de 47400000 dólares, y otras maquinarias por el mismo monto (Ministerio de Agricultura y CORFo, 1954, p. 20). El objetivo del primer periodo fue logrado con holgura: se importó un promedio anual de 2068 tractores, destacando 3333 unidades del año 1955 (véase gráfica 1). A diferencia del periodo anterior, entre 1958 y 1961 el promedio anual efectivo de importación de tractores resultó ser significativamente inferior al planificado (1 190 unidades). Considerando ambos periodos, la meta establecida en el plan estuvo relativamente cerca de cumplirse (13 034 unidades importadas de las 14840 proyectadas).

Como se aprecia en la gráfica 1, la internación de equipos se caracterizó por la inestabilidad, relacionada con los problemas financieros y cambiarios por los que atravesó el país desde mediados de siglo, lo que re-

${ }^{8}$ Ese mismo año el Decreto del Ministerio de Hacienda núm. 11.206 estableció un listado de maquinarias para internación libre de derechos. Apenas un par de años después, el decreto del Ministerio de Hacienda núm. 2277 (1952), liberaba de derechos e impuestos siempre que se acreditara mediante certificado CORFO que estaban las maquinarias destinadas exclusivamente a fines agrícolas.

${ }^{9}$ Los agentes importadores en Chile a fines de la década de 1940 eran: Compañía Distribuidora Nacional, Salinas y Fabres, Compañía de Petróleos de Chile, Gildemeister y Compañía Limitada, Saavedra Benard y Compañía, Guarello y Hantzke Limitada, Sociedad Importadora del Pacífico Limitada, Importadora Maquinaria Agrícola Sociedad Anónima, quienes comercializaban productos de 23 fabricantes extranjeros (Lorenzini, 1949, p. 39).

${ }^{10}$ Se plantea un papel potencial de la industria metalúrgica nacional en la sustitución de los repuestos. De hecho, el informe sugirió la instalación de una planta para la armaduría y montaje de tractores y otros equipos agrícolas, lo que se planteó como materia bajo estudio en el plan de 1954 . 


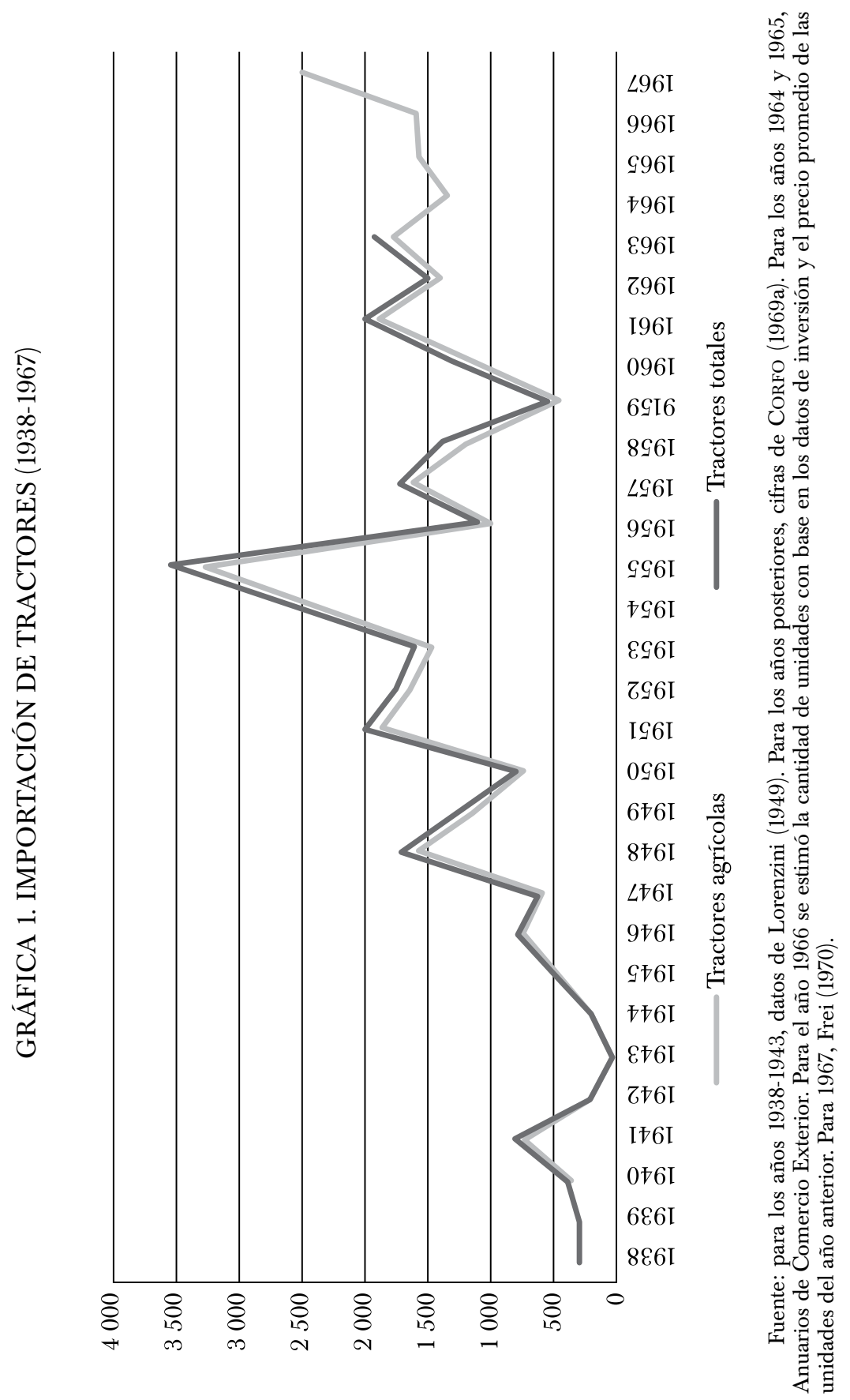


percutía en el presupuesto y las prioridades del Estado, especialmente tras la crisis de 1957 (Sunkel, 2011). En aquellos casos, los grandes y medianos productores podían acudir a créditos del Banco del Estado (Camhi, 1978). En 1960 la CORFO reinició los créditos para la importación de maquinaria, aunque mantuvo la irregularidad.

A mediados de la década de 1960, la mecanización pasó a tener un papel secundario dentro de los planes modernizadores, tal como se estableció en el Plan de Desarrollo Agropecuario 1965-1980 de la Oficina de Planificación Agrícola (ODEPA): "En la actual etapa de desarrollo de Chile sólo se deberá admitir la continuación de la tendencia natural que ha tenido la mecanización en los últimos años, orientándola de preferencia hacia aquellas inversiones imprescindibles para mejorar los rendimientos por unidad de superficie [...]. De esta manera, la política estatal no estimulará una mecanización exagerada e indiscriminada de la agricultura" (ODEPA, 1970, p. 43).

El temor de perjudicar el empleo agrícola, y por lo tanto, crear las condiciones para acelerar la migración campo-ciudad, sumado a resultados insatisfactorios en los incrementos productivos y la mayor organización política y sindical campesina, significaron un cambio en las prioridades del sector (Garrido, 1988).

\section{AVANCES EN LA MECANIZACIÓN}

A mediados de la década de 1960, y tras casi tres décadas de fomento estatal, la mecanización fue perdiendo relevancia en la agenda de política agrícola, saturada por las reivindicaciones y presiones en torno a la reforma agraria. ${ }^{11}$ A continuación se presenta la evolución del parque disponible en Chile, considerando tractores y otras maquinarias.

\section{Tractores}

La evolución de la cantidad de tractores utilizados en el país queda expresada en los distintos censos agropecuarios del periodo, cifras en las que se consideran unidades en funcionamiento con distintos grados de uso. El

\footnotetext{
${ }^{11}$ La reforma agraria en Chile tuvo dos fases. La primera, durante el gobierno de Jorge Alessandri (1958-1964), de modestos alcances y abarcando principalmente tierras fiscales. La segunda fase, ocurrida en los gobiernos de Eduardo Frei Montalva (1964-1970) y Salvador Allende (19701973), de amplios alcances (6 400000 hectáreas) (Garrido, 1988).
} 
parque tuvo incrementos muy significativos, con una tasa de crecimiento entre 1936 y 1976 de $2110 \%$ (véase cuadro 1).

Ahora bien, se debe profundizar en las diferencias territoriales en estas tasas. Para poder comparar el periodo completo, las provincias existentes en 1944 fueron agrupadas según las regiones creadas en 1974. Para el año 1944 la mayor cantidad de unidades se concentró en las regiones de la zona central (Valparaíso, Metropolitana, O’Higgins y Maule) y en la zona sur (Bío Bío, La Araucanía y Los Lagos) (véase mapa 1). Debido a que la zona central fue el núcleo histórico de la producción agroganadera del país, es natural su mayor disponibilidad de tractores. Por el contrario, la mayor parte de la zona sur fue de incorporación tardía, derivada del despojo a comunidades indígenas y posterior colonización de inmigrantes europeos desde fines del siglo XIX (Bengoa, 2014). Tras tres décadas, las regiones de la zona sur mostraron los mayores incrementos (junto a la región metropolitana).

Una posible explicación de la mayor mecanización en el sur es la que plantea Santana (2006). Desde comienzos de la década de 1930, el Estado intervino el mercado agrícola al fijar precios y establecer un poder de compra, creando para ello la Junta de Exportación Agrícola (Ley núm. 4912, 1930), ${ }^{12}$ transformada en 1942 en el Instituto de Economía Agraria ${ }^{13}$ y luego en 1960 en el Instituto Nacional de Comercio. ${ }^{14}$ Dentro de sus principales atribuciones se encontraban: fijar cuotas de exportación e importación, establecer primas por transporte, fijar precios del trigo, la harina y el pan, comprar y vender trigo y sus derivados u otros productos agrícolas, construir frigoríficos, fomentar la formación de cooperativas, entre otras (Gómez, 1944). El objetivo inicial era sostener al debilitado sector agrícola -en el contexto de la crisis mundial- asegurando un piso mínimo de compra. Luego, a fines de esa década, se convirtió en un mecanismo con el cual asegurar alimentos básicos a bajo costo para los trabajadores urbanos y de segmentación productiva por localización (centro-sur) (Santana, 2006).

${ }^{12}$ Dependiente del Ministerio de Agricultura. A partir de 1939 se le concede autonomía y patrimonio propio. El peso de los intereses de los grandes productores queda claro en su conformación. De los nueve miembros, cuatro eran designados por el presidente de la república y los otros cinco a partir de ternas presentadas por distintas asociaciones empresariales: la Sociedad Nacional de Agricultura; la Sociedad Agrícola del Sur y la Sociedad Cooperativa Agrícola de Temuco; la Sociedad Agrícola y Ganadera de Osorno; el Directorio de la Caja de Crédito Agrario, y por el Comité Central de Asociaciones de Molineros (Ley 5.034, 1934). Como señala una memoria de la época: "La composición de este Consejo o Junta ha sido objeto de acerbas críticas y aun se ha tratado de variarla en diversas oportunidades, mediante mociones presentadas al Congreso, incluyendo representantes de entidades obreras y de empleados" (Gómez, 1944, p. 11).

${ }^{13}$ Concentró las facultades de la Junta de Exportación Agrícola y el Consejo de Fertilizantes (véase Decreto con Fuerza de Ley 2851 de 1942).

${ }^{14}$ Fusión del Instituto de Economía Agrícola y el Instituto de Comercio Exterior (Decreto con Fuerza de Ley 274, 1960). 


\section{CUADRO 1. TRACTORES EN USO (UNIDADES)}

$\begin{array}{cc}\text { Año } & \text { Número de tractores } \\ 1930 & 660 \\ 1936 & 1557 \\ 1955 & 14177 \\ 1965 & 22307 \\ 1976 & 34407\end{array}$

Fuente: Dirección General de Estadísticas $(1933,1938,1953)$ e Instituto Nacional de Estadísticas $(1970,1981)$.

Durante los gobierno radicales (1938-1952), la influencia de los grandes productores del sur en el principal partido de gobierno (Partido Radical) les permitió obtener el establecimiento de precios diferenciados por distancia, manteniendo con ello la competitividad frente a sus pares del centro, afectados en menor medida por los costos de transporte (Santana, 2006). La propia localización de las oficinas de compra estatales favoreció a los productores del sur. No obstante, desde mediados de la década de 1950 esas condiciones favorables fueron modificadas, fijándose un precio de compra en el área de consumo principal (Santiago), en vez del área de producción principal (sur), con lo cual los costos de transporte y las múltiples intermediaciones ${ }^{15}$ restaron competitividad a estos últimos (Santana, 2006).

Ahora bien, desde el punto de vista del tamaño de las explotaciones, como era de esperar, fueron los grandes propietarios quienes incorporaron en mayor medida la fuerza mecánica, especialmente de los tractores de mayor potencia. En 1975-1976 en promedio en el país se contó con un tractor por cada nueve propiedades, pero mientras en las de menor tamaño era cada 125 explotaciones, en las mayores todas contaban con uno (véase cuadro 2). En la práctica, estas cifras minusvaloran el uso del tractor, por la posibilidad de arriendo. ${ }^{16}$ De hecho, las explotaciones con uso bajo arriendo fueron $50 \%$ más que las que contaban con tractor propio. En cuanto a

${ }^{15}$ Diferentes fuentes estiman entre seis y nueve intermediarios antes de la venta al detalle, obteniendo en torno a dos tercios del valor pagado por el consumidor. Véanse Astorga e Hidalgo (1966) y Fletschner (1971) en Santana (2006).

${ }^{16}$ A mediados de siglo, $11.6 \%$ de las propiedades trabajaba con maquinaria arrendada (Censo Agrícola 1955-1956). Además, se deben considerar aquellas en posesión de propietarios de explotaciones sin tierras, quienes en el censo agrícola de 1975-1976 contaban con 95 tractores, presumiblemente disponibles para arriendo. 


\section{MAPA 1. EVOLUCIÓN REGIONAL DEL PARQUE DE TRACTORES} (1944-1976)
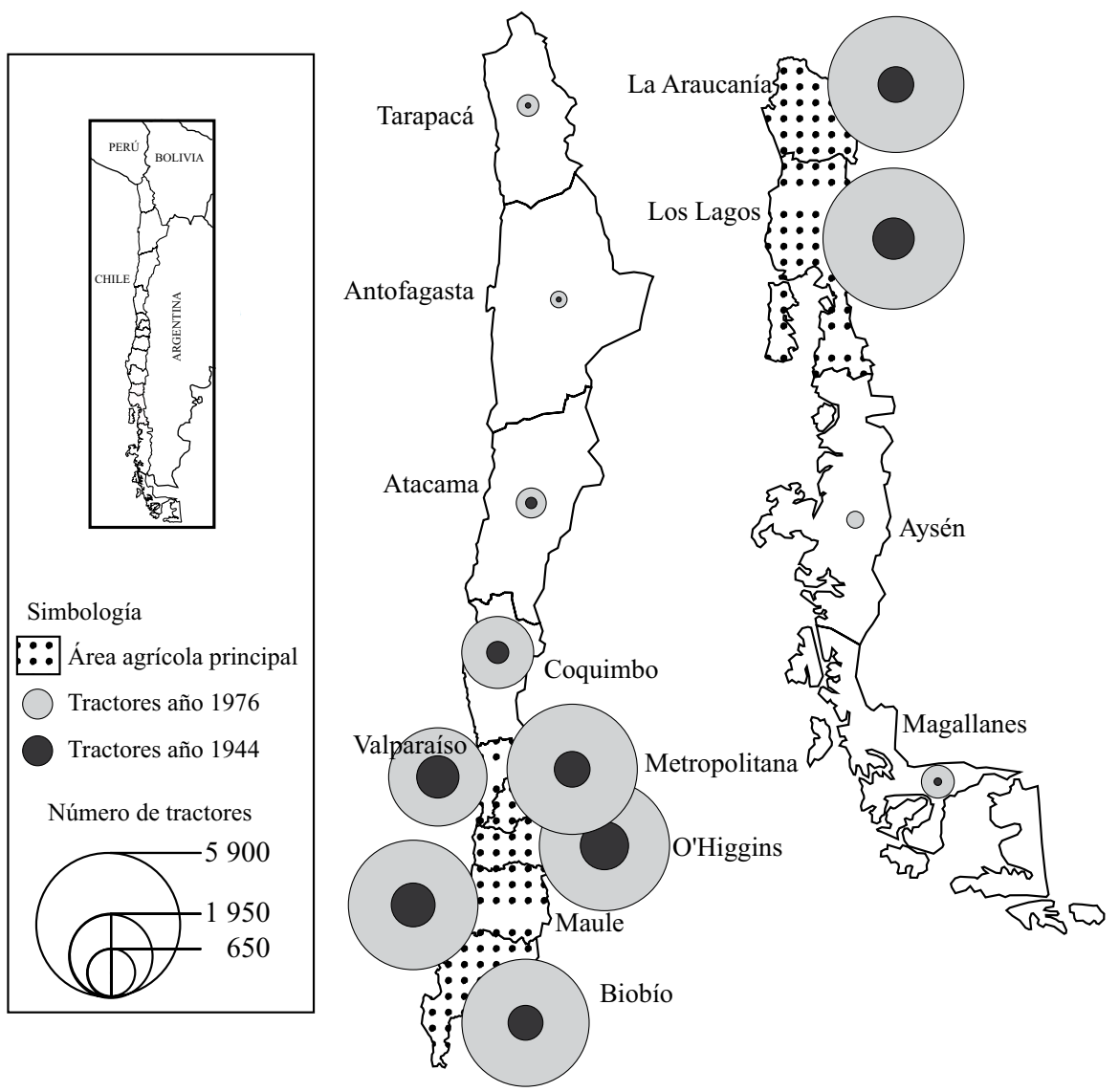

Fuente: elaboración propia con base en Empadronamiento CoRfo 1944, reproducido por Lorenzini (1949), y V Censo Agropecuario 1975-1976. 


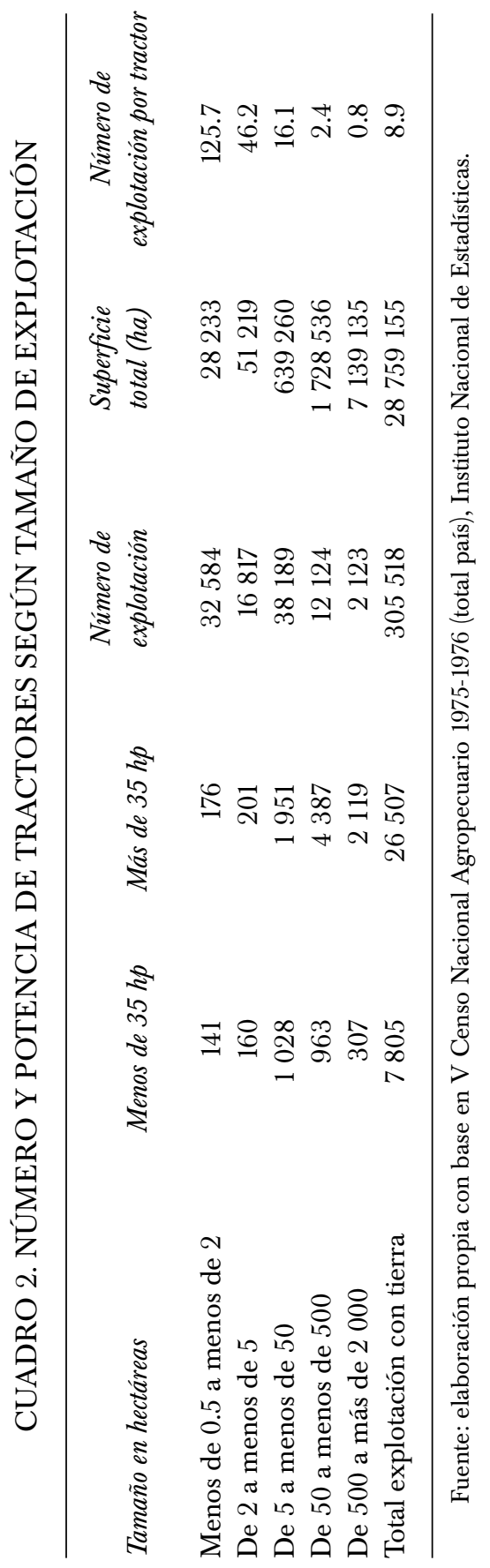


las cosechadoras y trilladoras fijas, las explotaciones que las utilizaban bajo arriendo eran casi siete veces más que las que contaban con la propiedad de estas (Censo Agropecuario, 1975-1976).

\section{Otras maquinarias}

A diferencia de los tractores, analizar otro tipo de maquinaria se dificulta por la poca consistencia en los registros y categorías entre los respectivos censos agropecuarios, ya sea por la agrupación de tipos de maquinaria o por la inclusión o exclusión variable de ellas. No obstante, para algunos periodos la información es útil. Considerando los quinquenios 1946-1950 y 1951-1955, se observa que las tasas de crecimiento porcentual de arados y rastras, sembradoras y automotrices son considerables, mientras que la importación de trilladoras, cosechadoras y sembradoras disminuyó, lo que parece dar cuenta de un uso ya extendido (véase cuadro 3).

A través del censo de 1955-1956 podemos ver la distribución territorial de trilladoras y cosechadoras y arados de fierro. ${ }^{17}$ Para analizar de manera algo más precisa, se ha considerado el área de mayor vocación agrícola (zonas centro y sur), en la cual se concentran 97\% de las trilladoras y arados de fierro y $92 \%$ de las cosechadoras del total de país. Al igual que en el caso de los tractores, se han agrupado según las regiones creadas en 1974, de manera de facilitar la comparación. Las cantidades en cursivas marcan las regiones por sobre el promedio del grupo (véase cuadro 4). En el caso de los arados de fierro, su uso es extendido y su distribución relativamente homogénea entre zonas del centro y del sur del país. Por el contrario, en el caso de las cosechadoras y las trilladoras, salvo en Santiago, se encuentran mayoritariamente presentes en la zona sur. Respecto a estas últimas, además, la disminución en el número de unidades importadas arriba señalada da cuenta de una cobertura extendida.

En comparación con los tractores, la adopción de trilladoras y cosechadoras fue menos homogénea territorialmente. Si bien diferencias en el tipo de suelo pueden ser un factor a considerar, llama la atención que sea en el Valle Central, plano y sin mayores problemas de inundación, donde se adoptaron en menor medida estas maquinarias.

Otra manera de analizar la incorporación de fuerza mecánica es a través del uso de pulverizadoras. Si bien el uso de estas estaba masificado, en su mayoría eran manuales y, a diferencia de los tractores, eran de fabrica-

\footnotetext{
${ }^{17}$ Por su simpleza puede ser considerada una incorporación tecnológica básica que además reemplaza una de uso tradicional (arado de palo), por lo tanto, es útil para conocer los grados de modernización en explotaciones de propietarios con bajo capital.
} 


\section{CUADRO 3. IMPORTACIÓN DE MAQUINARIA QUINQUENAL}

\begin{tabular}{lccc}
\hline Maquinaria & $\begin{array}{c}\text { Promedio } \\
1946-1950\end{array}$ & $\begin{array}{c}\text { Promedio } \\
1951-1955\end{array}$ & $\begin{array}{c}\text { Crecimiento } \\
\text { (porcentaje) }\end{array}$ \\
Arados y rastras & 940 & & \\
Automotrices & 110 & 2983 & 17 \\
Enfardadoras & 103 & 230 & 72 \\
Trilladoras & 84 & 177 & -48 \\
Cosechadoras & 146 & 78 & -47 \\
Sembradoras & 305 & 838 & 175 \\
Segadoras & 670 & 548 & -18 \\
\hline
\end{tabular}

Fuente: elaboración propia con base en Ministerio de Agricultura (1957).

\section{CUADRO 4. PARQUE DE ARADOS, COSECHADORAS Y TRILLADORAS}

\begin{tabular}{lccc}
\hline & Arados de fierro & Cosechadoras & Trilladoras \\
Valparaíso & 9335 & 117 & \\
Metropolitana & 14636 & 233 & 312 \\
O’Higgins & 18884 & 165 & 463 \\
Maule & 32983 & 255 & 578 \\
Bío Bío & 37506 & 350 & 945 \\
La Araucanía & 43064 & 676 & 1102 \\
Los Lagos & 29726 & 1384 & 1408 \\
Total país & 192881 & 3449 & 5211 \\
\hline
\end{tabular}

Fuente: Censo Agropecuario, 1955-1956.

ción nacional, de manera cuasi monopólicamente por la empresa Parada S. A. La incorporación de equipos manuales tuvo un crecimiento notable a fines de la década de 1960. Por el contrario, la incorporación de pulverizadoras mecánicas se mantuvo baja de manera estable (véase gráfica 2).

En síntesis, durante los 30 años de políticas de fomento a la mecanización agrícola se lograron incrementos importantes en el stock disponible, especialmente en tractores, arados, rastras, automotrices y sembradoras. 


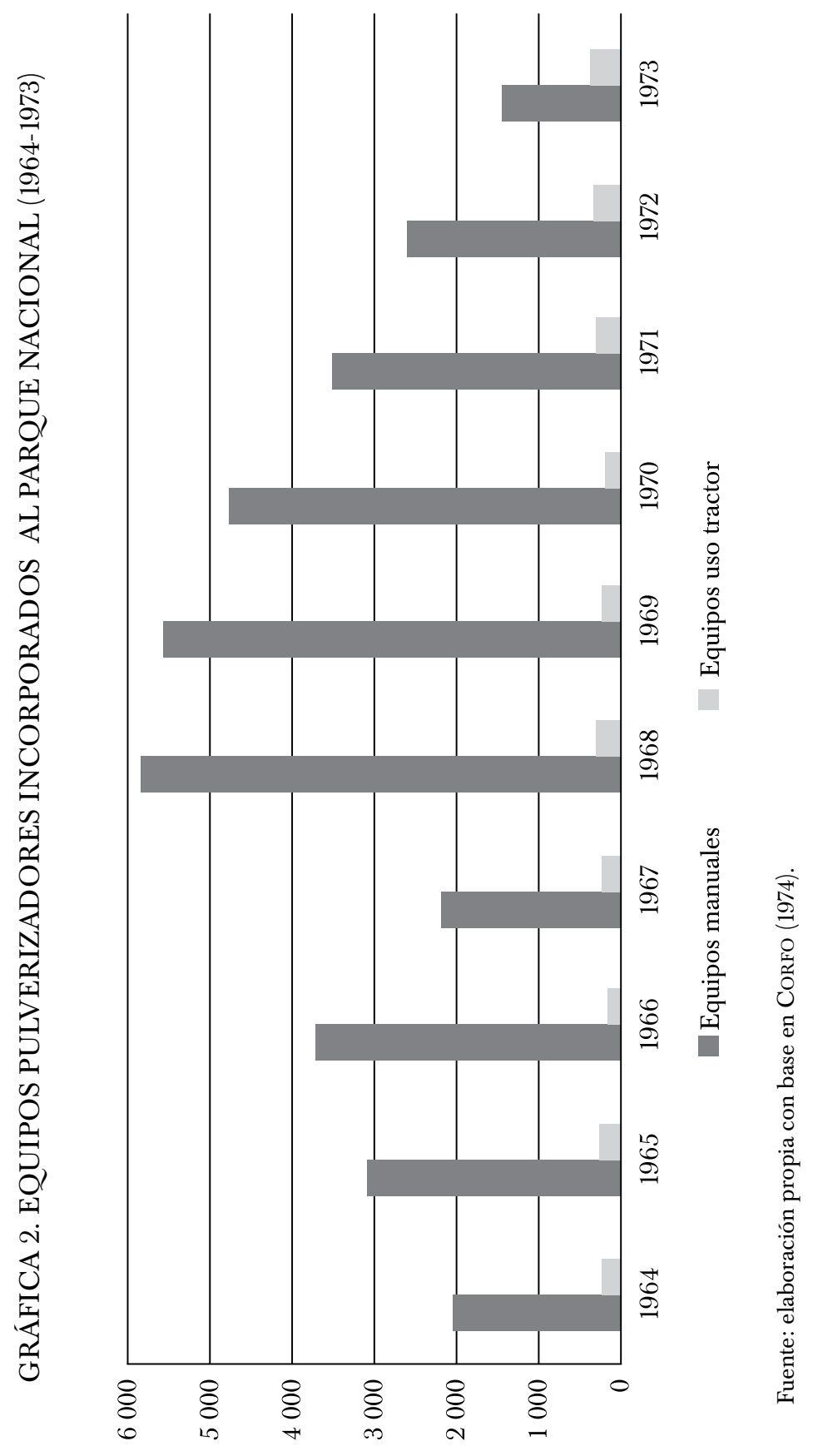




\section{Operarios}

El incremento de maquinaria significó una mayor demanda de personal calificado para su manejo, la cual no resultó cubierta. El grado de escolaridad en los campos era bajo, y las oportunidades de formación limitadas. Sumado a lo anterior, modificaciones institucionales previas resultaron adversas, mientras que las medidas adoptadas con posterioridad fueron limitadas en su impacto. Es así como hasta 1927 existió en el Ministerio de Agricultura un Servicio de Enseñanza y Fomento Agrícola, formado por un área de Enseñanza Industrial de Hombres y una de Enseñanza Profesional Femenina, contando en su último año con 288 funcionarios (casi la mitad del ministerio ese año). Luego, en 1931, fue reemplazado por el Servicio de Enseñanza Agrícola, de menor tamaño que el anterior, aunque más específico en sus funciones de capacitación, el cual tuvo vigencia hasta 1943 , contando con una planta promedio de 65 personas, aunque con fuertes variaciones de un año a otro (Urzúa y García, 1971, pp. 108-110). Años más tarde, cuando el problema se tornó más acentuado, se buscó revertirlo a través de breves cursos impartidos por militares a lo largo del país, los cuales tuvieron un impacto muy limitado debido a la baja matrícula de estudiantes (CORFO, 1969a).

Como ha sido planteado, los costos asociados al uso de maquinaria restringieron el acceso a los productores de explotaciones con bajo capital disponible. En ese contexto, el papel del Servicio de Equipos Agrícolas Mecanizados (SEAM) resultó determinante para las explotaciones pequeñas y medianas en buena parte del país, gracias al servicio de trabajos con maquinaria agrícola a precios subvencionados. Creado al interior de la CORFO, el SEAM se convirtió en la principal institución relacionada con la mecanización agrícola en Chile.

\section{Servicio de Equipos Agrícolas Mecanizados (SEAm): EL ESTADO EN DESPLIEGUE}

\section{Estructura y orgánica}

Sus antecedentes se encuentran en la creación en 1942 de la División de Equipos Aradores, sección especial dependiente del Departamento de Agricultura de la CoRfo. Durante esta etapa, sus labores consistían en la preparación de suelos, recolección de productos y promoción de maquinaria en terreno. Con el objetivo de permitir el acceso a los pequeños productores, el SEAM orientó sus labores hacia el arriendo de maquinaria pesada para funciones de habilitación de suelo por destronque, nivelación de 
potreros, estanques para riego, etc. (CORFO, 1969a). Una década después, el SEAM logra la autonomía jurídica, manteniendo aportes de capital de la CORFO. Sus objetivos fueron: "La explotación y comercio de maquinaria agrícola, mediante la producción, adquisición y venta de maquinarias; la ejecución por cuenta propia o ajena de labores con equipos mecanizados, y el saneamiento y drenaje de predios agrícolas" (Decreto con Fuerza de Ley 381, 1953).

A partir de entonces, la estructura del SEAM se compuso de un presidente y cinco directores, con duración en el puesto de tres años y posibilidad de reelección (y remoción) por parte de sus respectivos mandantes. Su planta administrativa se dividía en una planta directiva (un gerente general y los jefes de los departamentos comercial, productivo, legal y técnico); tres jefes de sección (contabilidad, auditoría y personal); una secretaría general, un inspector productivo y jefe de la estación de servicio. Contaba además con una planta productiva (empleados de las agencias) ${ }^{18} \mathrm{y}$ una planta administrativa (personal de las oficinas centrales de Santiago) ${ }^{19}$ (Contraloría General de la República, 1954). Posteriormente, esa estructura fue parcialmente modificada en el Decreto con Fuerza de Ley número 7 (1968), ampliándose las funciones hacia la capacitación e investigación.

\section{Composición}

En líneas generales, el SEAM en su fase de maduración (década de 1960) estaba compuesto por unas 1000 personas (80\% obreros y $20 \%$ empleados), distribuidas territorialmente en agencias, en número variable según las condiciones de cada época y el grado de demanda de los territorios. Para 1964 existían las de Ovalle (antes, Agencia Coquimbo), Rancagua, Talca, Chillán, Los Ángeles, Temuco y Osorno, habiendo sido suprimidas o anexadas a otras las correspondientes a las ciudades de San Fernando, Curicó y Tranques (1959) y luego Valdivia (1962). Posteriormente, se abrieron nuevas agencias en Antofagasta, Coyhaique, Arica, Iquique y Castro, es decir, avanzando hacia los extremos del país, zonas de baja vocación agraria. A pesar de esta marcada presencia territorial, el funcionamiento del SEAM se caracterizó por la centralización de gran parte de las decisiones en la oficina central de Santiago.

\footnotetext{
${ }^{18}$ Como esquema general, cada una de ellas cuenta con un agente, un subagente, un secretario, un mecánico de campo, un jefe de taller, un bodeguero, un auxiliar, un chofer mecánico y un chofer, más una cincuentena o más de obreros.

${ }^{19}$ En Santiago también se encuentra la estación de servicios donde se realizan las reparaciones más complejas y el almacén de repuestos.
} 
Las agencias debían relacionarse con una gran cantidad de órganos, lo cual dejaba abierto el riesgo de descoordinación, como efectivamente ocurrió. Sólo considerando las instituciones dentro de la CORFO relacionadas con la mecanización, podemos encontrar varios órganos, de distinta jerarquía (gerencia, división, departamento y sección), y no necesariamente dentro de la misma cadena de mando, abordando elementos específicos de la política de mecanización: Gerencia Agrícola ${ }^{20}$ División de Agricultura, ${ }^{21}$ Departamento de Mecanización Agrícola ${ }^{22}$ Departamento de Créditos Agrícolas ${ }^{23}$ y Sección Maquinaria Agrícola ${ }^{24}$ (CORFO, 1969c). Considerando el resto de los ministerios y órganos relacionados, correspondían a 21 instituciones en la materia deficientemente coordinadas (ODEPA, 1970). Olivares (1962), en su análisis sobre la agencia de O’Higgins, hizo especial hincapié en ese aspecto, señalando:

No existe, a nivel territorial, una buena coordinación entre las instituciones del Sector Público Agrícola. Esta coordinación sólo se logrará por una reformulación de la estructura de organización de la mayoría de ellas. [...] Sus métodos de planificar las actividades no favorecen un sistema de operación enmarcado por Planes Regionales de Desarrollo Agropecuario. De ahí que la posibilidad de operar en esta forma está condicionada a una reformulación de las estructuras de organización, a la creación de sistemas regionales de planificación y de organismos específicamente destinados a la coordinación regional (p. 1).

\section{Funciones}

En 1959 el Servicio contó con 221 tractores de diverso tipo, con sus arados, rastras y equipos correspondientes, lo que, considerando el censo agropecuario más próximo (1956), representaban en torno a 2\% del parque naracional.

${ }^{20}$ Una de sus funciones es promover el desarrollo agrícola a través de una mecanización

${ }^{21}$ Tiene como objetivo mantener las relaciones institucionales con el Banco Central, el Banco del Estado, la Corporación de Reforma Agraria, el Instituto de Desarrollo Agropecuario, Dirección de Industria y Comercio, la Empresa Nacional de Semillas y la Empresa Nacional de Frigoríficos, además del propio SEAM.

${ }^{22}$ Sus funciones son la preparación del diagnóstico de la maquinaria agrícola; estudio y evaluación del plan nacional de mecanización agrícola; mantener un registro de importadores de maquinaria agrícola; investigar tipo de maquinaria que se debe importar.

${ }^{23}$ Otorga créditos a los productores agrícolas en sentido general.

${ }^{24}$ Dentro del Departamento de Créditos Agrícolas, tiene como función otorgar créditos con el fin de adquirir maquinaria agrícola para todo el país; resolver los problemas de prórroga; control de los convenios con los importadores de maquinaria agrícola y con los fabricantes de repuestos de dichas maquinarias, y control de las importaciones, principalmente a través de la oficina de Nueva York. 
cional. Ese año contó además con 115 automotrices. Si bien la dotación señalada no es numéricamente significativa, su uso intensivo permite esperar un impacto de cierta relevancia. En el periodo 1953-1964 en promedio se trabajaron 262575 horas anuales, mostrándose los menores niveles durante el trienio $1959-9161 .^{25}$ En el mismo periodo las funciones se distribuyeron en labores de suelo $(42.4 \%)$, faenas pesadas agrícolas $(28.2 \%)$, trabajos pesados no agrícolas $(15.8 \%)$ y de cosecha (13.5\%) (CORFO, 1969a).

Desde un punto de vista territorial -a partir de los ingresos generados-, las agencias de mayor dinamismo fueron las de la zona sur del país (Osorno, Temuco y Chillán) y la de Santiago, lugar de la oficina central (véase gráfica 3).

Ahora bien, desde el punto de vista de los costos, emerge un elemento importante. Paulatinamente los gastos de la institución tuvieron un incremento, que a mediados de la década de 1960 se convirtió en déficit. La principal causa de esta situación fue el aumento de los sueldos y salarios, especialmente a comienzos de la década (véase gráfica 4). De manera agregada los empleados obtenían $41.3 \%$ de las remuneraciones -siendo aproximadamente $20 \%$ de los trabajadores-, mientras que los obreros representaban $58.7 \%-80 \%$ de las personas-. La participación de los empleados en el total de remuneraciones fue considerada excesiva por entonces (Franceschini, 1965).

Se conjugan varios elementos para explicar esta alza en los salarios. En primer lugar, el contexto inflacionario del periodo (Sunkel, 2011), que los funcionarios públicos sortearon generalmente de mejor forma debido a su capacidad de presión política. Relacionado con ello, a diferencia de otras empresas estatales que actuaban con autonomía de las consideraciones partidarias, el SEAM fue un órgano reconocido como cuota política del Partido Radical ${ }^{26}$ (directivos), aunque con temprano control sindical del Partido Comunista (Torrejón, 2010).

A partir de los acuerdos logrados entre la empresa y la Asociación Nacional de Obreros del Servicio de Equipos Agrícolas Mecanizados, fundada en 1952, es posible establecer la gran capacidad para lograr incrementos salariales y otros beneficios, como asignaciones escolares y familiares. ${ }^{27}$ Hacia fines de la década de 1960 la evaluación de la gerencia agrícola de la CORFo era crítica en cuanto al funcionamiento técnico, administrativo

\footnotetext{
${ }^{25}$ El índice de horas trabajadas por trienio respecto a 1953 fue: 1953-1955 (105\%), 1956-1958 (90\%), 1959-1961 (67\%), 1962-1964 (154\%) (CORFO, 1969a).

${ }^{26}$ Entrevista personal a Rolando Chateauneuf, académico en economía agrícola y ex alto funcionario de CORFO, 24 de marzo de 2015.

${ }^{27}$ En los doce años considerados, se produjeron nueve acuerdos respecto a mejoras en las condiciones laborales (varias de ellas incluyeron tanto a obreros como empleados). Véase folleto de la Asociación Nacional de Obreros del SEAM (1964).
} 

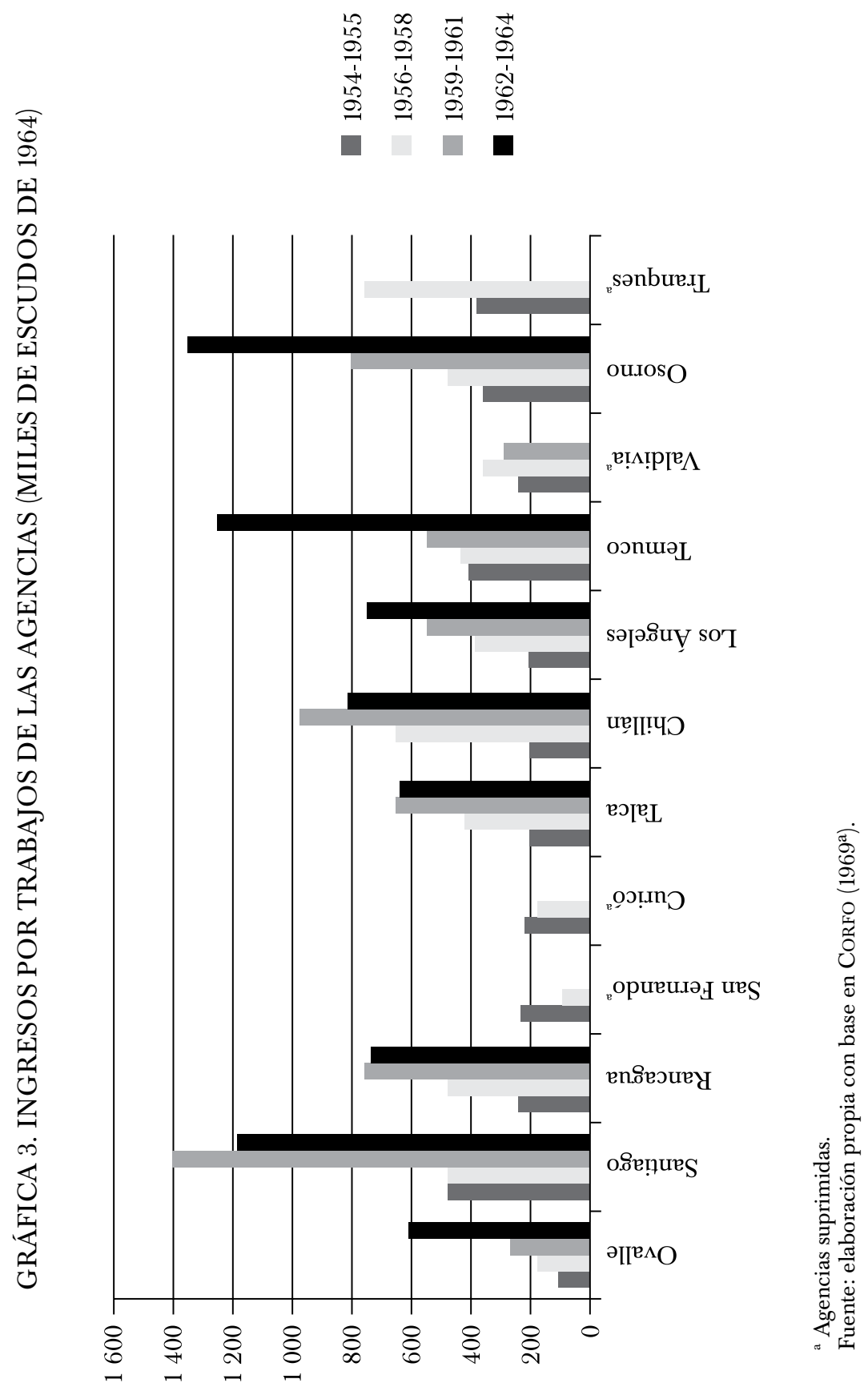


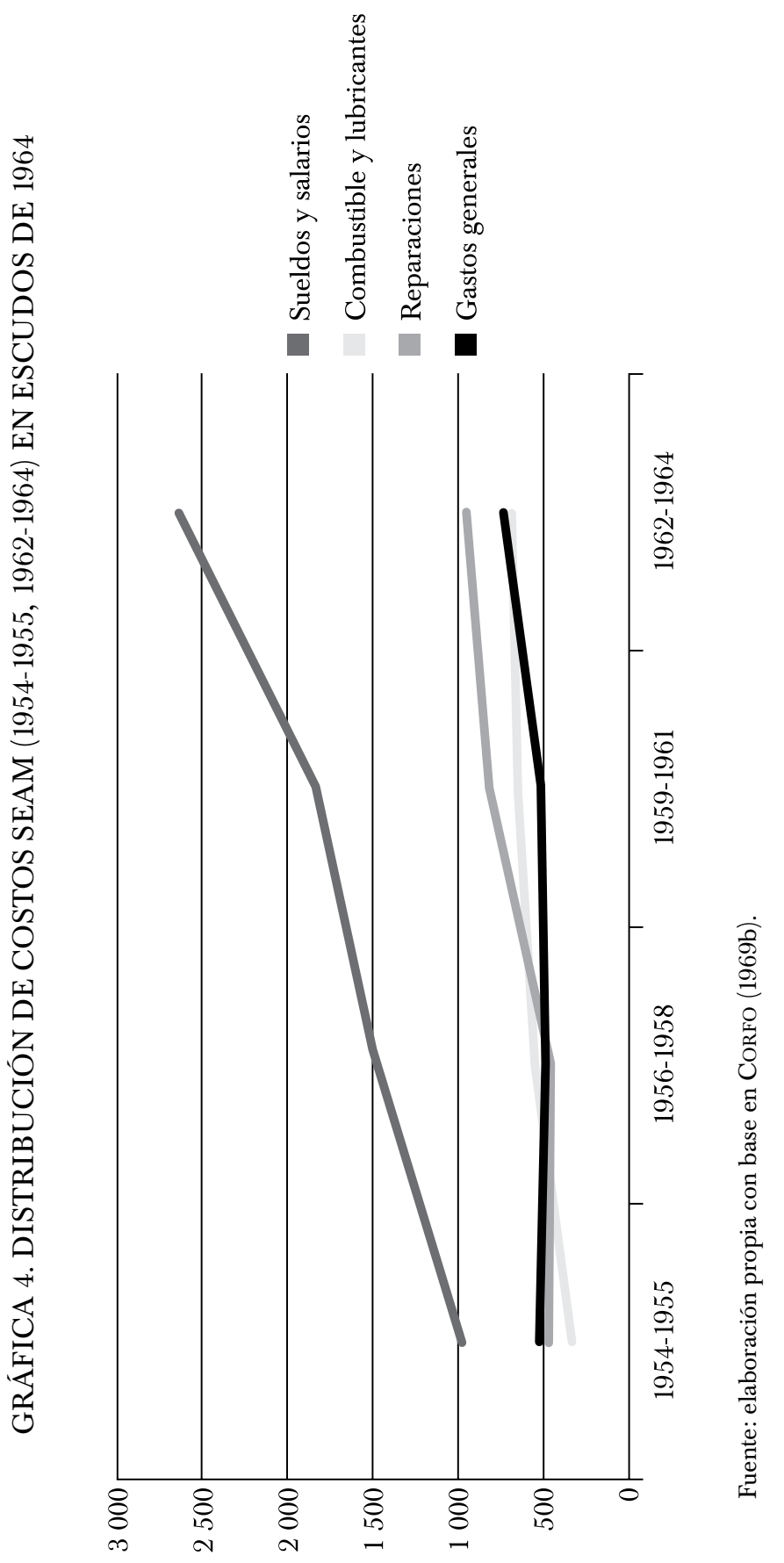


y financiero. El tenor de las expresiones de la gerencia agrícola por entonces permite derivar otros problemas en su funcionamiento, además de los financieros, proponiendo -entre otras medidas- la creación de un grupo de asistencia técnica al SEAM y seminarios para elevar las competencias del personal (Gerencia Agrícola, 1969, p. 37). Otros informes plantearon conclusiones similares en términos de la necesidad de reformarlo administrativamente (Departamento de Administración en Reforma Agraria, 1966; Franceschini, 1965; ODEPA, 1970). A medida del avance de la reforma agraria y la incorporación de las nuevas unidades productivas reformadas, el universo de potenciales beneficiaros del SEAM se fue incrementando, debilitándose su impacto en el conjunto. Debido al golpe de Estado de 1973 el funcionamiento de la institución fue congelado, no concretándose los planes de reforma. Legalmente el servicio tuvo existencia hasta el año 1979 (Decreto Ley 2572), aunque en la práctica esos últimos seis años se destinaron a la venta de los activos disponibles.

\section{CONCLusiones}

Tras tres décadas de fomento estatal, los avances en la incorporación de maquinaria a las faenas agrícolas en Chile fueron relevantes en lo cuantitativo. Sin embargo, el déficit de alimentos internos no pudo ser revertido, manteniéndose el agrícola como un sector rezagado de la economía. Varios elementos explican lo sucedido. En primer lugar, la distribución de la maquinaria se caracterizó por la concentración en las unidades de mayor tamaño, debido a los altos costos asociados a su internación y funcionamiento. Para lo primero, el Estado decretó la exención arancelaria y créditos blandos, principalmente a través de la CORFO (pequeños productores) y del Banco del Estado (grandes productores), aunque no siempre de manera focalizada. Para lo segundo, se adoptaron medidas de bajo impacto, como los cursos para preparar tractoristas y la exención arancelaria para los repuestos (medida restringida y tardía). Las instituciones públicas del área agrícola carecieron además de una estrategia global de mecanización, actuando en forma descoordinada, e incluso superpuesta, ralentizando los procesos y restando eficiencia en el gasto público.

Frente a ello, el SEAM buscó ser un puente para la mecanización de las unidades menores a través del arriendo de maquinarias o servicios, cumpliendo una función importante en segmentos específicos, pero relativamente limitado en su alcance global por contar con apenas $2 \%$ de la maquinaria del país. Su despliegue territorial resultó importante en el fomento de la mecanización en algunas provincias (especialmente en la zona centro-sur), a pesar de su manejo excesivamente centralizado y los proble- 
mas administrativos y financieros, especialmente en los últimos años. La inestabilidad en sus inversiones, reflejo de los vaivenes de la economía del país, fue otro factor que limitó sus alcances. Finalmente, los problemas en capital humano, estrategias de inversión desacertadas (stocks excesivos de repuestos), los fuertes incrementos salariales y el deterioro del clima laboral por causa de la polarización política, restaron capacidad al SEAM para asumir una participación más protagónica en la modernización agrícola de pequeños y medianos productores.

\section{LISTA DE REFERENCIAS}

Ahumada, J. (1958). En vez de la miseria. Santiago: Editorial del Pacífico.

Almonacid, F. (2009). La agricultura chilena discriminada (1910-1960): una mirada de las políticas estatales y el desarrollo sectorial desde el sur. Madrid: Colección América Csic. Asociación Nacional de Obreros del Seam (1964). Estatutos I [folleto]. Santiago: Imprenta Horizonte.

Avendaño, O. (2014). Los partidos frente a la cuestión agraria en Chile, 1967-1973. Política. Revista de Ciencia Política, 52(1), 93-122.

BARSKY, O. (1994). Transformaciones productivas y sociales en la pampa argentina. En M. Twomey y A. Helwege (comps.), Modernización y estancamiento. La agricultura latinoamericana en los años noventa (pp. 67-92). México: Fondo de Cultura Económica.

BENGOA, J. (1990). Haciendas y campesinos. Historial social de la agricultura chilena [t. II.]. Santiago: Ediciones Sur.

BengOA, J. (2014). Mapuche, colonos y el Estado Nacional. Santiago: Catalonia.

CAMHI, R. (1978). Análisis del crédito agrícola en Chile en el periodo 1960-1976: un intento de cuantificación del subsidio vía crédito entregado al sector (Tesis de licenciatura). Universidad de Chile, Santiago, Chile.

CARrière, J. (1981). Landowners and politics in Chile. A study of the Sociedad Nacional de Agricultura 1932-1970. Ámsterdam: Centro de Estudios y Documentación Latinoamericanos.

CEPeda, O. y GitLi, E. (1985). Producción de tractores y transferencia de tecnología en México: el T-25 de origen soviético. Comercio Exterior, 35(8), 799-805.

Contraloría General de la RePÚblica (1954). Recopilación de los decretos con fuerza de ley [t. XLI, vol. 2]. Santiago: Imprenta del Pacífico.

Corporación de Fomento de la Producción [Corfo] (1940). Plan de acción inmediata para la agricultura y explotaciones afines. Santiago: Imprenta y Enc. Letelier.

CorPoración de Fomento de LA Producción [CORFo] (1969a). Mecanización agrícola en Chile: diagnóstico a 1963 [t. I. Áreas agrícolas; existencia de maquinaria]. Santiago: Dirección de Planificación Corfo. 
CORPORACIÓN DE FOMENTO DE LA PRODUCCIÓN [CORFO] (1969b). Mecanización agrícola en Chile: diagnóstico a 1963 [t. II. Política arancelaria; análisis de precios]. Santiago: Dirección de Planificación CORFo.

Corporación de Fomento de la PRODUCCión [CORFo] (1969c). Manual preliminar de organización y funciones. Santiago: Vicepresidencia Ejecutiva Grupo de Trabajo de Presupuestos por Programas, Santiago.

Corporación de Fomento de la Producción [Corfo] (1974). Estudio de mercado nacional de pulverizadores agrícolas. Santiago: División de Industrias Mecánicas.

CORPORACIÓN PARA LA REFORMa AgRARIA [CORA] (1970). Reforma agraria chilena, 1965-1970. Santiago: Corporación de la Reforma Agraria.

Correa, S. (2005). Con las riendas del poder. La derecha chilena en el siglo XX. Santiago: Editorial Sudamericana.

Departamento de Administración en Reforma Agraria (1966). Organización, planificación y coordinación de las instituciones del sector público agrícola de Chile, a nivel de terreno. Informe de investigación. [Borrador de circulación restringida]. Santiago: Ministerio de Agricultura.

Dirección General de Estadística (1933). I Censo Agropecuario 1929-1930. Santiago: Dirección General de Estadística.

Dirección General de Estadística (1938). II Agricultura 1935-1936: Censo. Santiago: Dirección General de Estadística.

Dirección General de Estadística (1953). XII Censo General de Población y I de Vivienda año 1952. Santiago: Dirección General de Estadística.

FERNÁNDEZ, L. (1999). Estado, sociedad rural e innovación tecnológica en la agricultura. Los cambios en torno a 1900. Studia Historia. Historia Contemporánea, 17, 67-103.

Figallo, F. y Vattuone, M. E. (1989). Tecnología: el lado oscuro de la reforma agraria. Debate Agrario, 7, 103-125.

Franceschini, A. (1965). Análisis crítico del funcionamiento del Servicio de Equipos Agrícolas Mecanizados (SEAM) (Tesis de licenciatura). Universidad de Chile, Chile.

Frei, E. (1970). Lo que Chile está realizando 1965-1970. Santiago: Editorial Zig-Zag.

GARrido, J. (ed.) (1988). Historia de la reforma agraria en Chile. Santiago: Editorial Universitaria.

Gerencia Agrícola (1969). Memoria de gerencia agrícola. Santiago: Oficina de Programación y Estudios.

Gómez, J. (1944). La Junta de Exportación Agrícola (hoy Instituto de Economía Agrícola). Memoria de prueba Licenciatura en Ciencias Jurídicas y Sociales de la Universidad de Chile. Santiago: Imprenta El Imparcial.

Gómez, J. C. (2004). La frontera de la democracia: el derecho de propiedad en Chile, 19251973. Santiago: Lom Editores.

Instituto Nacional de Estadística (1970). IV Censo Nacional Agropecuario: año agrícola 1964-1965. Santiago: Instituto Nacional de Estadísticas.

Instituto Nacional de Estadística (1981). V Censo Nacional Agropecuario 1975-1976, total país. Santiago: Instituto Nacional de Estadísticas. 
Letelier, E. (1967). Manual de fertilizantes para Chile. Santiago: Editorial del Pacífico.

Lorenzini, E. (1949). Mecanización agrícola (memoria inédita de Licenciatura en Ciencias Jurídicas y Sociales). Universidad de Chile: Chile.

MamalaKis, M. (1976). The growth and structure of the Chilean economy. New Haven: Yale University Press.

MC BRIDE, J. (1938). Chile: su tierra y su gente. Santiago: Universidad de Chile.

Meller, P. (1998). Un siglo de economía política chilena (1890-1990). Santiago: CiePlan.

Millar, R. y Fernández, J. (2008). Políticas agrarias en Chile: 1932-1958, Boletín de la Academia Chilena de la Historia, año 74 (117), 407-464.

Ministerio de AGRicultura (1957). La agricultura chilena en el quinquenio 1951-1955. Santiago: Departamento de Economía Agraria.

Ministerio De AgriCultura y CORFo (1954). Plan de desarrollo agrícola y de transportes. Santiago: Gobierno de Chile.

Moulian, T. (2006). Fracturas. De Pedro Aguirre Cerda a Salvador Allende (1938-1973). Santiago: Lom Editores.

Muñoz, O. y Arriagada, A. M. (1977). Orígenes políticos y económicos del Estado empresarial en Chile. Colección Estudios Cieplan, núm.17. Santiago: Corporación de Estudios para Latinoamérica.

Negrete, J. C. (2014). Rural poverty and agricultural mechanisation policies in Mexico. Journal of Agriculture and Environmental Sciences, 3(1), 46-66.

Oficina de Planificación Agrícola [Odepa] (1970), Plan de Desarrollo Agropecuario 1965-1980 (2a. ed.). Santiago: Imprenta Camilo Henríquez.

Olivares, J. (1962). Estudio de la mecanización agrícola en la Provincia de O’Higgins (año agrícola 1958-1959). Santiago: Universidad de Chile/Ministerio de Agricultura.

Ortega, L. (coord.) (1989). Corporación de Fomento de la Producción: 50 años de realizaciones 1939-1989. Santiago: Universidad de Santiago de Chile.

PInTO, A. (1959). Chile, un caso de desarrollo frustrado. Santiago: Editorial Universitaria.

RACCANELlo, M. (2012). Surcando una nueva industrialización. El estado empresario en la fabricación de maquinaria agrícola (1952-1961), en III Congreso Latinoamericano de Historia Económica y XXIIIJornadas de Historia Económica, 23-27 de octubre, San Carlos de Bariloche, Argentina.

RoBles, C. (2003). Expansión y transformación de la agricultura en una economía exportadora. La transición al capitalismo agrario en Chile (1850-1930). Historia Agraria, 29, 45-80.

Robles, C. (2009). Controlando la mano invisible: la Sociedad Nacional de Agricultura y el mercado de maquinaria agrícola (1889-1922). Historia, 42(1), 203-233.

Rodríguez Weber, J. (2013). Economía política de la distribución del ingreso rural en Chile durante la decadencia de la Hacienda, 1935-1971. Revista Uruguaya de Historia Económica, III(3), pp. 33-62.

SANTANA, R. (2006). Agricultura chilena en el siglo XX: contextos, actores y espacios agrícolas. Santiago: Centro de Estudios Regionales y Políticas Públicas de la Universidad de lo Lagos/Dirección de Bibliotecas, Archivos y Museos de Chile. 
Sunkel, O. (2011). El presente como historia. Dos siglos de cambio y frustración en Chile. Santiago: Editorial Catalonia.

Torrejón, F. (2010). Relatos de un obrero. Vida de un comunista. Santiago: Editorial Luciérnaga.

Urzúa G. y A. García (1971). Diagnóstico de la burocracia chilena. Santiago: Editorial Jurídica.

\section{BIBLIOGRAFÍA}

Servicio Nacional de Estadísticas y Censos (1959). III Censo Nacional Agrícola Ganadero. 1955-1956. Santiago: Servicio Nacional de Estadísticas y Censos. 